Revue Revue de l'histoire des religions

de Ihistoire des religions

Théologie politique et sciences sociales : autour d'Erik Peterson (1890-1960)

Christian-Bernard AMPHoux et Jacqueline AssAëL (éd.), Philologie et Nouveau Testament. Principes de traduction et d'interprétation critique

Aix-en-Provence, Presses Universitaires de Provence (« Héritages méditerranéens »), 2018

Matthieu Cassin

\title{
OpenEdition
}

Journals

Édition électronique

URL : https://journals.openedition.org/rhr/11304

DOI : $10.4000 /$ rhr. 11304

ISSN : 2105-2573

Éditeur

Armand Colin

Édition imprimée

Date de publication : 1 septembre 2021

Pagination : 552-555

ISBN : 978-2-200-93377-7

ISSN : 0035-1423

Référence électronique

Matthieu Cassin, « Christian-Bernard Amphoux et Jacqueline Assä̈L (éd.), Philologie et Nouveau

Testament. Principes de traduction et d'interprétation critique », Revue de l'histoire des religions [En ligne],

3 | 2021, mis en ligne le 01 septembre 2021, consulté le 04 octobre 2021. URL : http://

journals.openedition.org/rhr/11304; DOI : https://doi.org/10.4000/rhr.11304

Ce document a été généré automatiquement le 4 octobre 2021.

Tous droits réservés 


\section{Christian-Bernard AMPHOUX et Jacqueline AssAëL (éd.), Philologie et Nouveau Testament. Principes de traduction et d'interprétation critique}

Aix-en-Provence, Presses Universitaires de Provence (« Héritages méditerranéens »), 2018

Matthieu Cassin

\section{RÉFÉRENCE}

Christian-Bernard Amphoux et Jacqueline AssAëL (éd.), Philologie et Nouveau Testament. Principes de traduction et d'interprétation critique, Aix-en-Provence, Presses Universitaires de Provence (« Héritages méditerranéens »), 2018, 328 p., $24 \mathrm{~cm}, 32 €$, ISBN 979-10-320-0189-9.

1 Le texte biblique a été l'un des premiers à susciter la réflexion sur les modalités de sa transmission et de son édition, tant du fait de son importance religieuse intrinsèque que des modalités pour le moins complexes de sa transmission. Parmi les textes de langue grecque, le Nouveau Testament a joué un rôle central dans ces études, au même titre qu'Homère. Pourtant, depuis bien des années, pour ne pas dire des décennies, les philologues ont dans leur grande majorité abandonné le texte biblique, au profit des seuls exégètes de profession. Les ponts sont largement rompus entre étude des textes grecs, chrétiens ou non, et étude du Nouveau Testament; la Septante, au contraire, grâce au travail de Marguerite Harl et de ses élèves, a connu un sort différent et est l'objet, depuis les années 1980 au moins, d'un travail mené par des hellénistes. La séparation, en France, des Facultés de théologie du reste de l'Université, depuis la suppression de la Faculté de théologie de l'Université de Paris en 1885, n'a certes pas aidé à l'instauration d'un dialogue entre exégètes et philologues. À défaut d'un tel 
dialogue, le présent ouvrage collectif, issu d'un colloque tenu à Aix-en-Provence en 2014, proposait à des philologues, non à des exégètes, d'aborder des points particuliers du Nouveau Testament, en particulier des points textuels difficiles, soit directement, soit à travers leurs interprètes antiques et médiévaux. L'objectif affiché était de revenir au texte, en mettant à distance - mais sans les ignorer - les cadres propres aux systèmes doctrinaux.

2 Le livre s'ouvre par une introduction de J. Assaël, qui brosse un rapide tableau des relations entre philologie et herméneutique dans le cas du Nouveau Testament, en tenant compte aussi des positions de l'Église catholique ou de philosophes. La première partie concerne une série de variantes textuelles particulières ou l'approche des variantes dans une tradition d'interprétation particulière. C'est ce dernier cas qui constitue la première contribution, due à Gilbert Dahan, et qui porte sur la critique textuelle dans les correctoires du $\mathrm{XIII}^{\mathrm{e}} \mathrm{s}$., ces recueils de notes critiques. Ch.B. Amphoux s'attaque ensuite aux éditions imprimées du Nouveau Testament, dont il dénonce la mise en avant exclusive du texte égyptien, alexandrin, au détriment des autres formes textuelles; l'auteur conteste le primat donné à ce type de texte et plaide en faveur d'une nouvelle édition, tripartite, présentant à la fois le texte alexandrin, le texte byzantin et le texte occidental, en privilégiant dans chaque cas un manuscrit choisi. Laurent Pinchard se penche sur deux passages matthéens (Mt 26, 55 et 28,8) dans le Codex de Bèze, qui révèlent non des leçons harmonisantes mais des échos vétérotestamentaires. David Pastorelli étudie la célèbre variante de Jn 1, 18 (Dieu ou Fils monogène), en recourant aux témoignages anciens pour dater et localiser l'origine des leçons. Étienne Nodet consacre son étude à deux variantes supposées marcionites (Lc 7, 18 et Ga 2,1) - ce travail est paru sans avoir pu tenir compte d'études récentes et importantes sur le Nouveau Testament de Marcion (voir par exemple Apocrypha 27, 2016, p. 199-212); sans entrer dans une discussion détaillée, on notera au moins que l'une des affirmations finales, selon laquelle le concile de Nicée aurait été « le moteur d'une révision générale du NT » est dépourvue de tout élément de preuve.

3 Une seconde partie aborde la critique textuelle à partir des commentaires patristiques. La philologie est ici au second degré et étudie les travaux textuels anciens et les témoignages patristiques pour mieux atteindre le texte néotestamentaire. Jean Reynard confronte tradition directe et indirecte sur la question de la finale de Marc, lieu problématique si souvent étudié; le parcours est riche et très suggestif, même s'il aurait pu être plus enrichi et nuancé encore sur certains points, par exemple dans l'étude des citations bibliques de l'adversaire païen de Macarios de Magnésie et de ses sources (voir diverses contributions dans S. Morlet [éd.], Le traité de Porphyre contre les chrétiens : un siècle de recherches, nouvelles questions. Actes du colloque international organisé les 8 et 9 septembre 2009 à l'Université de Paris IV - Sorbonne ["Études augustiniennes ", Série Antiquité 190], Paris 2011). Christian Boudignon reprend le dossier du Notre Père dans Luc et en particulier de la demande de l'Esprit; ce verset est en effet connu, par Grégoire de Nysse puis par Évagre le Pontique et Maxime le Confesseur, ainsi que par deux manuscrits néotestamentaires, sous une forme variante, «que vienne ton Esprit saint (sur nous) et qu'il nous purifie ». L'auteur, à bon droit, écarte en la matière le fantôme de Marcion. Régis Burnet s'arrête au nom du dixième apôtre, Thaddée ou Lebbée.

4 La troisième partie concerne non plus la critique textuelle, mais la critique littéraire, toujours à partir des textes patristiques. J. Assaël étudie 2 Co $4,10-15$, autour des 


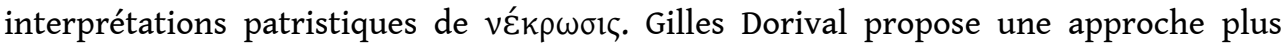
globale des lieux problématiques relevés par les exégètes anciens (ici limités à Clément d'Alexandrie, Julius Africanus, Origène, Eusèbe de Césarée) dans le Nouveau Testament, autour de quelques exemples (péricope du jeune homme riche, généalogies de Jésus, début et fin des évangiles). Laurence Mellerin étudie les différentes formes et exégèses du logion sur le blasphème contre l'Esprit (Mt 12,31-32) - cette contribution aurait aussi pu trouver place dans la partie précédente. Pierre Molinié choisit un tout autre angle, avec l'examen du phénomène des récapitulations bibliques, de la reprise résumée de quelques versets commentés, afin d'en dégager les fonctions dans la prédication chrysostomienne. Jenny Read-Heimerdinger applique l'analyse du discours à l'étude des différences entre le texte des Actes dans le Vaticanus (B) et le Codex de Bèze (D) - on s'étonne d'ailleurs de la place de cette contribution, intéressante en elle-même, en une partie consacrée aux commentaires patristiques ; c'est d'ailleurs probablement le seul texte du volume qui vienne d'une spécialiste du Nouveau Testament, contrairement au projet d'ensemble.

5 La quatrième et dernière partie est consacrée aux exégèses médiévales - période déjà présente en ouverture du volume avec la contribution de G. Dahan. J. Casteigt traite du baptême et de la colombe dans le commentaire sur Jean d'Albert le Grand. Édouard Robberechts consacre son étude à la ligature d'Isaac entre Gn 22, 2 et Jc 2, 21 ainsi que He 11, 17 ; il confronte l'interprétation néo-testamentaire à celle de Rabbi Lévi ben Gershon (1288-1344). Ch.-B. Amphoux conclut l'ouvrage en étudiant le double mouvement de « déconstruction » (par quoi il entend les modifications d'un texte pour le faire entrer dans le corpus de référence) et de «reconstructions» (processus qui conduit à une reconfiguration d'ensemble afin de donner une unité satisfaisante au corpus), à partir de divers exemples : Jc, Jr et les Évangiles pour la déconstruction, et diverses études du volume pour les reconstructions. L'auteur conclut en opposant les reconstructions patristiques aux rédactions des textes néotestamentaires, qui ne seraient, elles, accessibles que par les traditions textuelles. L'opposition finale paraît un peu forcée, au contraire même de ce que montrent plusieurs des contributions, où les traditions textuelles - et non seulement interprétatives - peuvent être pour partie reconstruites à partir des témoignages patristiques.

6 L'approche d'ensemble de ce volume est tout à fait intéressante : ramener le regard des philologues, et non des seuls exégètes, sur le Nouveau Testament, afin de soumettre son texte et sa tradition textuelle et interprétative à une critique qui ne soit pas d'emblée orientée par une perspective doctrinale et/ou confessante. Plusieurs des contributions de l'ouvrage en montrent d'ailleurs la portée, tout autant que l'intérêt qu'il y a non seulement à prendre en compte le témoignage textuel des auteurs chrétiens des premiers siècles, mais aussi à le replacer dans le contexte interprétatif des auteurs concernés. La chose n'est pas nouvelle, mais une telle approche est féconde, à n'en pas douter - même si elle aurait sans doute parfois gagné à s'appuyer plus largement sur les travaux déjà menés, tant sur le texte biblique que sur les textes patristiques convoqués. On pourrait espérer qu'un tel ensemble fasse renaître plus d'intérêt, chez les spécialistes des textes grecs chrétiens ou profanes, pour le texte grec de l'Ancien et du Nouveau Testaments et leurs interprétations anciennes, sans les laisser à la seule approche des exégètes et théologiens - mais sans écarter non plus la dimension religieuse et les traditions chrétiennes et juives dans lesquelles ces textes se sont développés. 


\section{AUTEURS}

\section{MATTHIEU CASSIN}

Institut de recherche et d'histoire des textes

(CNRS, UPR 841), Paris. 